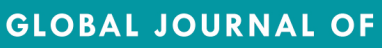 \\ Community Psychology Practice
}

\section{PROMOTING COMMUNITY PRACTICE FOR SOCIAL BENEFIT}

Self help groups in a city of Tuscany: Reconstruction of the second generation model of work for professionals and services.

Petrini, F., Vannucchi, S., Miraglia Raineri A., \& Meringol, P.

University of Florence

Keywords: self help, community psychology, gjcpp

Petrini, F. PhD student, Psychology Dept., University of Florence.

(fausto.petrini@unifi.it)

Vannucchi, S., trainee student, Psychology Dept., University of Florence.

Miraglia Raineri A., trainee student, Psychology Dept., University of Florence.

Meringolo P., Professor of Community Psychology, Psychology Dept., University of Florence.

Recommended citation: Petrini, F., Vannucchi, S., Miraglia Raineri A., \& Meringol, P. (2012). Self-help groups in a city of Tuscany: Reconstruction of the second generation model of work for professionals and services. Global Journal of Community Psychology Practice, 2(3), 1-12. Retrieved Day/Month/Year, from (http://www.gjcpp.org/). 


\title{
Self help groups in a city of Tuscany: reconstruction of the second generation model of work for professionals and services.
}

\author{
Petrini, F. PhD student, Psychology Dept., University of Florence. (fausto.petrini@unifi.it) \\ Vannucchi, S., trainee student, Psychology Dept., University of Florence. \\ Miraglia Raineri A., trainee student, Psychology Dept., University of Florence. \\ Meringolo P., Professor of Community Psychology, Psychology Dept., University of Florence.
}

\section{INTRODUCTION}

This study is part of a more extensive project aimed to investigate the effectiveness of self-help group participation in improving quality of life in mental disease. The study is taking place in the Tuscany Region, in Italy. In the first qualitative step of analysis researchers are interested in describing the specific features of the psychiatric self-help movement in Tuscany, comparing different realities, networks, kind of groups. Therefore, our aim is to collect exhaustive information to describe how self-help system work in different provinces at the present moment. The implementation of groups for psychiatric problems is quite young in Italy. Because of a lack of specific regulation in the directives of the Italian health care system, every local service has implemented groups differently, sometimes enhancing, sometimes dismissing them. Prato, near Florence, is one of the more interesting context for the birth of psychiatric self-help movement in the region: public health services improved groups since early 90's, it was one of the first self-help reality linked to services in the entire region. Now we are in a "second generation" of professionals, and the original meaning of groups seems to be transformed, sometimes misunderstood. Our objectives of study head us toward an in depth analysis of self-help phenomenon in Prato.

\section{BACKGROUND}

Community psychology has long served as the more suitable context for self-help research and practice. Due to its purposes of emancipation and empowerment, the practice of self help in mental health have been acquiring more and more relevance in community research. Brown points out how "this special issue builds on community psychology's historical foundation in community mental health by exploring the burgeoning practice of mental health self-help. Since the founding of community psychology, the community mental health system has increasingly come to embrace the values of self-direction and mutual support embodied by mental health self-help initiatives" (Brown, Shepherd, Merkle, Wituk \& Meissen, 2008).

Empowerment and social support are two of the most important theoretical perspectives that are commonly used to describe the benefits from mental health self-help (Helgeson and Gottlieb 2000; Holter et al. 2004; Maton and Salem 1995; Nelson et al. 2001; Segal et al. 1993). The empowerment perspective explains how involvement in organizational leadership can promote recovery, whereas the social support literature explains how social involvement can impact recovery.

Despite this, how participants benefit from mental health self-help is not fully understood (Helgeson and Gottlieb 2000). Self help groups are a complex phenomenon indeed: it represent a challenge to the researcher seeking to explain their effectiveness. Multiple factors seems to be involved in the self help psychological and social process, many researchers tried to find evidences on different levels of their equations (Bloch and Crouch 1985; Kyrouz and Humphreys 1996; Maton and Salem 1995).

From another point of view, qualitative methods provide an interesting way to study effectiveness from the participants perspective. A large literature of surveys, qualitative studies, and first-person accounts attests to the subjective benefits of mutual help groups (see, for example Borkman, 1999; Humphreys, 2000; Leavy, 2000). Qualitative analysis is also a key element for sited research, best way to deeply describe a specific territorial context.

Finn, Bishop \& Sparrow (2009) in a qualitative study about GROW organization, underline multifactorial processes of change across the individual level, the group level and the program/community level. This is also an useful example of how qualitative research can highlight the historical perspective of participation in self help group.

By an ethnographic study Laws (2009) shows how the shared values in the group context of self help could even influence the participants perception of therapeutic places. 
Finally, the study of Salem, Reischl \& Randall (2008) about the partnership between Schizophrenics Anonymous and Mental Health Association in Michigan shows the reciprocal changes in values and practice in an historical perspective.

Some of the most important findings in this field indicate the uniqueness of self-help/mutual aid groups in the weaving of emotional and practical information and support. Overall, it appears that self help groups offer something that cannot be replicated in professional- user relations. Therefore, self-help activity is not a substitute for professional services, but rather, it serves a different purpose for members, and the findings suggest that it may lead to more confident and appropriate use of existing services, as well as challenging them. Most of the qualitative studies on mutual support groups countries demonstrated a variety of benefits of group participation reported by the group participants, such as increased knowledge about the illness and its treatment, reduced burden and distress, and enhanced coping ability and social support.

\section{AIMS}

The aim of this contribution is to provide an in depth analysis of the whole self-help reality in a town in Tuscany (Italy). In particular, we are interested in evaluate different positive aspects depending on kind of group, kind of interviewee, historical changing in the meaning of participation by professionals.

\section{METHOD}

\section{PARTICIPANTS}

The present contribution focus attention on the qualitative data coming from professionals, key informants and patients involved in the 4 groups of the town. Twelve patients were interviewed during two focus groups, The six facilitators and 4 key informants, coming from institutional and medical context, were interviewed singularly. Three of the four groups in the town are directly linked with the mental health service: facilitators are nurses in their working hours, members are sent by psychiatrist, etc. The last group is consumer-run and it was born in the service too, but it became independent (some years ago). Therefore, the four groups were linked one another in their past experience, but they are working in a very different way at the present moment. Also the three groups conducted by mental health professionals are very different in number and kind of activity, time for discussion, etc.

\section{INSTRUMENTS}

Semi-structured interviews and focus-group aimed to investigate the following focus areas:

- Relationship between participation in the self-help group and quality of life;

- $\quad$ Effects of participation on frequency of medical consultations and on objective indicators of health (emergency services, prescribed psychotropic drugs...);

- $\quad$ Effects of participation on relationship with general practitioner and/or psychiatrist/psychotherapist in charge.

\section{DATA ANALYSIS}

All the sessions were registered and transcribed verbatim. Data were analyzed with a qualitative approach inspired by a Grounded Theory perspective (Glaser and Strauss, 1967; Charmaz, 2003), by means of the Atlas.ti software for qualitative analysis. In the first step of analysis, data from facilitators and members (all the people directly involved in group participation) and data from key informants (not directly involved) were analyzed separately. Comparison between the two source of data was performed in the second step of analysis to understand how intentions coming from professionals and managers have been translated in practical activities. Further interesting data emerge from the comparison between the three groups linked to the mental health service and the independent one.

\section{RESULTS}

\section{History: "Democratic Psychiatry" and the closedown of mental hospital}

In Italy Mental hospitals were closed since 1978 by means of a groundbreaking low inspired to community approach to health. This innovation was supported by a large social movement ("antipsychiatric movement") with supporters also in medical sectors ("democratic Psychiatry"). Results show how this inspiration is embedded in the values of the groups. Moreover, this kind of topics emerge also in the "first generation" of professionals (nurses and key informants) involved in the first group organization, in the late 80's. Democratic approach, empowerment of the patient, clearing of dependence and social advocacy are central categories in the analysis of key informants data:

\footnotetext{
"Basaglia...just some word about Basaglia's reform [for the closedown of mental hospitals], which also self help followed: the meaning...well... They put attention on the social aspects of psychiatric problems. Therefore, society was considered a sick society, and so society, to heal
} 
itself, must gave opportunity to keep conscious to people with problems... “"

(nurse, facilitator)

Most of the interviewees underline the progressive loss of these values in the new generation of professionals, more interested in economic benefits of self-help activities than in social functioning.

\section{Activities and discussion groups: integrated aspects or misrepresentation?}

An interesting issue seems to be the organization of activities (artistic activities, as poetry and theater sessions, or sport activity, etc.) to promote greater integration within the local community and to prevent stigma. Some groups perceive it as a key element, a more specific way to channel communication by different emotional modality. Some facilitators perceive activities as a misrepresentation of self-help methodologies instead:

"... at the same time, a series of activities started, in particular from nurses...Hence, they ask for a drawing teacher, they hold of a gymnastic teacher... and these things were much more liked, for example nurses like them very much. Self help groups could more like to physicians and psychologists, they think to "words groups", but to the ... you know, to practical professionals, activities like more. For example [...] the other head physician said "forget about self help! It is a nuisance!, lets bring them to play sports!" and sure it taken root, I mean ... [...] It sounds like: "I'll give you pills and gymnastic, just to let you metabolize it, instead self help is something different, it has different conditions, cause you have also to revise some mental health elements... "

(Key informant, physician and institutional promoter of self help groups)

\section{Effectiveness as quality of life, or just in terms of economic advantages?}

Both Key Informants and group members (Professionals and Patients) perceive advantages in improved quality of life, greater well-being, and mitigation of disease. They also report a lower use of "pills", fewer hospitalizations, a better socialization, which are thought as basic objectives for self-help groups.

"In the beginning there was an involvement of the mental service, and so a training for physicians too. Hence we were trying to prompt them to send patients... because... just because the consciousness of their problems could be useful for a better quality of life, and sometimes, why not, to avoid their problems, and it could be useful also for the whole society, because society could improve itself..."

(nurse, facilitator)

In particular, one of the key element for the selfhelp effectiveness seems to be a positive self redefinition of the patient's condition:

\section{"Because there are people that cant' understand things, than there are people who... you can understand each other, you know? That's the point. Today...everyone have a lot of nervous... a lot of agitation, so... it's not because they don't go to the doctor...but...I see them more nervous than me, so... what I'd like to say is... there is people who say it and people who not". \\ (group member)}

This can represent an advantage also for services, but sometimes it seems to be perceived just in economic terms by professionals, instead of therapeutic effectiveness. The relationship with services agree positive outcomes for both the service and the patients, about saving financial resources, avoiding unnecessary medical consultations or inappropriate use of psychiatric emergency services.

\section{The need for training of professionals in self- help culture}

Another relevant issue is the training about selfhelp culture for professionals and volunteers, that appears necessary to consumers for enhance positive outcomes. The first generation of professionals approach to self help meaning by means of specific training, international observation and participation to international conference. Same participants don't perceive the same interest in the new generation of professionals, underlining a future possible weakness of the intervention model.

\footnotetext{
"There is no many new nurses interested to these things... cause training for nurses today has become more technical, generalized... Social Psychiatry needs a completely different training instead... and it needs also of a different openness, because if you... You know, you must recognize that the psychiatric problem belong also to you, and then you can train yourself in a rewording way, cause you feel you are resolving also a problem of yours" (nurse, facilitator)
}

\section{Comparison between independent group and group linked to the service}


Both the professionals and the consumers not coming from the one independent group perceive it as more isolated and static:

“...It remains an independent group, but unfortunately not... They have been quite closing $e$ on themselves! Namely, this independence have driven them near the aridity, because they don't look for a relation with us, so..." (nurse, facilitator)

In actuality, the born of groups inside the service seems to be the cause of a strong reduction the independent group's attraction:

"We broke away from the service, there is no more agreement now, nurses don't attend the group any more, that is... also because in the meantime the service set up its own groups, and it was the reason of our...comparing with the first times...it was the reason of our crisis, cause, obviously, no more people were send to us, no more sending happens". (facilitator of the "independent group")

Even if independence from services seems to be an empowering element in literature, more observations in an ecologic perspective are needed. For example, few data try to explain how and why groups could compete for newcomers, seen as a "fuel" for vitality, and how this happens in presence of a selective service contribution.

\section{Service dependence and model of welfare}

From the opposite point of view, also other groups show some weaknesses in their link with services. The presence of professional operators, even if trained in self-help practice, set up a risk of dependence, minimizing empowerment possibilities:

" They couldn't make it by themselves! First of all, because, as I said, these groups were born from our initiative. In other words ... users, in every respect... they have already everything in every aspect, in their opinion. Almost everyone have...

INTERVIEWER: In other words, they find it as already existing...

Yeah, obviously! With low 180 [the low for the closedown of mental hospitals and the beginning to the democratic approach to psychiatry] they have all the facilitations, hence they have nothing to request. So it's just a proposal of us, which make us saying that they can stay better..."

(nurse, facilitator)

This element is well known in literature, but Italy have a very strong model of welfare, in which dependency culture is sometimes taken for granted.
This contextual value can hide risks not only to professionals, but also to consumers. Both key informants and patients define groups as "not separatist", meaning that no requirements is needed, no claim is embedded in the weaving of groups. In our opinion, service organization of groups could enhance the risk of a disempowering model of selfhelp, mitigating part of their value.

\section{CONCLUSION}

Obviously, results show great effort to effectiveness perception of group attendance. Groups seems effective to improve quality of life and well-being, through a better knowledge of problems and the sharing of experiences. They appear to perform psychological functions, such as perceived social support and increasing coping strategies, through the comparison with other members. Participants feel themselves increasing self-esteem and self-fulfillment. Far from invalidate these findings, we tried to highlight weaknesses and emergent questions. The comparison with other experiences, taken from different context and thanks to an ecological perspective, could enhance the effectiveness of self help movement in planning new experiences and managing relations with professional services.

\section{REFERENCES}

Bloch, S., \& Crouch, E. (1985). Therapeutic factors in group psychotherapy. Oxford: Oxford University Press.

Borkman, T. J. (1999). Understanding selfhelp/mutual aid: Experiential learning in the commons. New Brunswick, NJ: Rutgers University Press.

Brown, L.D., Shepherd, M.D., Merkle, E.C., Wituk, S.A., \& Meissen, G. (2008). Understanding how participation in a consumer-run organization relates to recovery. American Journal of Community Psychology, 42, 167-168.

Charmaz, K. (2003). Grounded Theory. In Smith, J. (Ed.) Qualitative Psychology: A practical guide to research methods. London: Sage publications.

Finn, L.D., Bishop, B.J., \& Sparrow, N. (2009). Capturing dynamic processes of change in GROW mutual help groups for mental health. American Journal of Community Psychology, 44, 305-315.

Glaser, B. G., \& Strauss, A. L. (1967). The discovery of grounded theory: Strategies for qualitative research. Chicago: Aldine.

Helgeson,V.S.,\&Gottlieb, B. H. (2000). Support groups. InS.Cohen,L. G. Underwood, \& B. H. Gottlieb (Eds.), Social support measurement 
and intervention: A guide for health and social scientists (pp. 221-245). Oxford, England: Oxford University Press.

Holter, M. C., Mowbray, C. T., Bellamy, C. D., MacFarlane, P., \& Dukarski, J. (2004). Critical ingredients of consumer run services: Results of a national survey. Community Mental Health Journal, 40(1), 47-63.

Humphreys, K. (2000). Community narratives and personal stories in Alcoholics Anonymous. Journal of Community Psychology, 28, 495-506.

Kyrouz, E. M., \& Humphreys, K. (1996). Do psychiatrically disabled people benefit from participation in self-help/mutual aid organizations? A research review. Community Psychologist, 29, 21-25.

Laws, J. (2009). Reworking therapeutic landscapes: The spatiality of an "alternative" self-help group. Social Science and Medicine. 69, 18271833.

Levy, L. (2000). Self-help groups. In J. Rappaport \& E. Seidman (Eds.), Handbook of community psychology (pp. 591-613). Dordrecht, Netherlands: Kluwer Academic Publishers.

Maton, M.I., \& Salem, D.A. (1995). Organizational characteristics of empowering community settings: A multiple case study approach. American Journal of Community Psychology, 23, 631-656.

Nelson, G., Lord, J., \& Ochocka, J. (2001). Shifting the paradigm in community mental health: Towards empowerment and community. Toronto, Canada: University of Toronto Press.

Salem, D.A., Reischl, T.M., \& Randall, K. W. (2008). The effect of professional partnership on the development of a mutual-help organization. American journal of community psychology, 42, 179-191.

Segal, S. P., Silverman, C., \& Temkin, T. (1993). Empowerment and self-help agency practice for people with mental disabilities. Social Work, 38, $705-712$. 